\title{
IN SITU SIMULATION OF CARDIAC ARREST
}

\author{
Michael Czekajlo ${ }^{1,2,3}$, Agata Dabrowska ${ }^{2,4}$ \\ ${ }^{1}$ Hunter Holmes McGuire VA Medical Center, Department of Surgery, Richmond, USA \\ ${ }^{2}$ Polish Society of Medical Simulation, Poland \\ ${ }^{3}$ Medical Simulation Centre, Lublin Medical University, Lublin, Poland \\ ${ }^{4}$ Department of Medical Rescue, Chapter of Emergency Medicine, Poznan University of Medical Sciences, Poznan, Poland
}

\begin{abstract}
In-hospital cardiac arrest is one of the most complicated events healthcare providers will manage in a hospital setting. Cardiac arrest also is a rare event compared to other clinical events managed on a routine basis, requiring deliberate preparation of staff to be able to have the knowledge, technical skills and teamwork necessary to manage such an event when it occurs.
\end{abstract}

Simulation-based education has been demonstrated to be an effective method of educating healthcare providers. More recently, hospital systems have begun using in situ simulation for the training of healthcare providers and identifying latent safety threats.

In situ simulation is defined as: "Taking place in the actual patient care setting/environment in an effort to achieve a high level of fidelity and realism; this training is particularly suitable for difficult work environments, due to space constraints or noise".

KEY WORDS: in-hospital cardiac arrest, resuscitation, education, in situ, medical simulation

Disaster Emerg Med J 2017; 2(3): 116-119

\section{INTRODUCTION}

In-hospital cardiac arrest is one of the most complicated events healthcare providers will manage in a hospital setting. Cardiac arrest also is a rare event compared to other clinical events managed on a routine basis, requiring deliberate preparation of staff to be able to have the knowledge, technical skills and teamwork necessary to manage such an event when it occurs.

Simulation-based education has been demonstrated to be an effective method of educating healthcare providers [1]. More recently, hospital systems have begun using in situ simulation for the training of healthcare providers and identifying latent safety threats $[2,3]$.

In situ simulation is defined as: "Taking place in the actual patient care setting/environment in an effort to achieve a high level of fidelity and realism; this training is particularly suitable for difficult work environments, due to space constraints or noise" [4].

Code Blue, or sudden cardiac arrest, in a MRI (magnetic resonance imaging) scanner is even more difficult to deal with and cardiac arrest in some others units and complicates management of the patient because of the additional safety precautions required due to the magnet in the MRI.

The Hunter Holmes McGuire VA Medical Center has two MRI scanners. One scanner is in the hospital building while the second unit is in a trailer outside of the building, approximately 15 metres away. The MRI staff are required to have annual training for a cardiac arrest in the MRI scanner.

This article describes the use of in situ simulation to identify code team readiness and latent safety threats in the MRI radiology suite. 


\section{METHOD}

In preparation for the simulation, two meetings were held to discuss the steps in the process. A discussion based on a Failure Modes and Effects Analysis was followed in identifying potential failure modes namely, failure causes and failure effects [5]. A process was established to ensure that no one involved in the simulation would accidently enter the MRI that was not screened. A safety review was also conducted to make sure non-MRI-approved equipment would access the MRI suite.

The chief of staff and other appropriate staff were informed that a mock "Code Blue" would be conducted in the MRI. The members of the Code Blue team were deliberately not informed to maintain fidelity of the scenario. Two simulation staff members were on site to inform the Code Blue members to treat this as a real "Code Blue" and to assist in maintaining fidelity of the scenario.

\section{OBJECTIVE}

To identify failure modes and failure causes in a Code Blue scenario occurring in the MRI trailer.

\section{SCENARIO}

A 65-year-old male with recent history of stroke was undergoing an MRI of brain. While in the scanner,

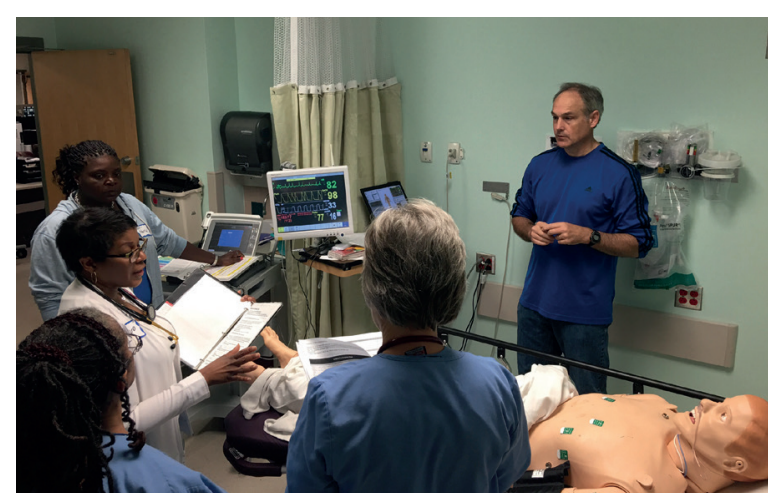

FIGURE 1. ER stroke sim training

he suffered a cardiac arrest and was found to be in a state of Ventricular Fibrillation (Fig. 1, Tab. 1).

\section{RESULTS}

A Laerdal 3G Human Patient Simulator, simulated crash cart, and video debriefing were utilized in order to gather data.

There is no validated tool for the assessment of RRT (rapid response team) or Code Blue teams. However, the literature does support the use of the "Mega code Checklist" by the American Heart Association. In order to assess this scenario, the event data from the simulator and video recorded data was used. In making our conclusions, we used the widely accepted guidelines of time to chest

Table 1. Scenario Progression
$\begin{gathered}\text { Time } \\ \text { (min/sec) }\end{gathered}$

*Event times were objectively recorded as they occurred by the SimMan $3 \mathrm{G}$ simulator ; ${ }^{* *} \mathrm{ED}$ - Emergency Department; ${ }^{* * *} \mathrm{ROSC}$ - return of spontaneous circulation 


\begin{tabular}{|c|c|c|}
\hline Critical Performance Steps & If done correctly & Comments \\
\hline \multicolumn{3}{|l|}{ Activation } \\
\hline \multicolumn{3}{|l|}{ Change in patient's condition identified } \\
\hline RRT/Code BLUE activated & - & $\begin{array}{l}\text { The MRI technician appropriately informed } \\
\text { the operator but was kept on the line } \\
\text { for confirmation for } 45 \text { seconds which } \\
\text { delayed activation and management }\end{array}$ \\
\hline \multicolumn{3}{|l|}{ VF/VT Pulseless } \\
\hline \multicolumn{3}{|l|}{ VF recognized } \\
\hline \multicolumn{3}{|l|}{ Cleared before Analyze and Shock } \\
\hline \multicolumn{3}{|l|}{ CPR immediately resumed after shocks } \\
\hline Appropriate airway management & - & $\begin{array}{l}\mathrm{BVM}^{*} \text { ventilation was performed but was } \\
\text { recorded as inadequate on the simulator }\end{array}$ \\
\hline Appropriate cycles of drug-rhythm check/shock-CPR & & $\begin{array}{l}\text { Second defibrillation was performed 1:28 } \\
\text { minutes after the first. Another cycle of } \\
\text { CPR should have been performed }\end{array}$ \\
\hline \multicolumn{3}{|l|}{ Appropriate drugs and doses administered } \\
\hline \multicolumn{3}{|l|}{ Post-Cardiac Arrest Care } \\
\hline \multicolumn{3}{|l|}{ ROSC identified } \\
\hline \multicolumn{3}{|l|}{$\begin{array}{l}\text { Ensured that BP and } 12 \text { lead ECG are performed, } 02 \text { saturation } \\
\text { is monitored, need for endotracheal intubation and waveform } \\
\text { capnography verbalized, and laboratory tests ordered }\end{array}$} \\
\hline Therapeutic hypothermia considered & N/A & \\
\hline
\end{tabular}

${ }^{*}$ BVM — big valve mask

compressions $<4$ minutes and time to defibrillations $<4$ min in Pulseless VF/NT (Tab. 2).

\section{DISCUSSION}

In situ simulation has been shown to be a useful method for resuscitation team training and for identifying potential patient safety threats in the hospital [6]. The use of in situ simulation is becoming common practice for training since it brings training to the healthcare provider in the environment they are working in on a daily basis. In situ simulation is grounded in adult learning theory.

In our pilot study of assessing an unannounced cardiac arrest in the MRI scanner, not only did we assess cardiac arrest preparation but it was done in a high-risk environment that could have severe consequences should a health care provider walk into an MRI scanner having metal on their person, or in the form of equipment introduced.

Overall, in the assessment of this scenario we found that the providers involved were aware of MRI safety risks and that the MRI personnel were adequately prepared to prevent personnel from walking into the MRI scanner. In assessing system issues of cardiac arrest management, we identified a significant system issue in the form of a 45 second delay in activating the code team due to communication with the hospital operator.

Another safety issue identified was transport from the MRI trailer to the hospital. In an actual cardiac arrest, it is ideal if compressions are started immediately. In this scenario, compressions were started 2:40 minutes following the arrest which meets the recommendation of $<4$ minutes. The recommendation of time to shock is $<4$ minutes and preferably $<3 \mathrm{~min}$. The first defibrillation in this scenario was performed at 06:37. The Laerdal $3 G$ manikin used in this scenario provides objective feedback to the quality of CPR skills and timing of interventions, including drug delivery and defibrillation. In this scenario the team members appropriately started all interventions and followed the VF/VT guidelines for management [7]. However, although bag valve mask ventilation was initiated, tidal volume on the simulator was identified as inadequate in order to maintain oxygenation, an issue which was not identified by the team. 
Our experience using in situ simulation identified gaps in knowledge, skills and logistics that are useful in targeting training and system redesign in order to improve patient safety in a similar way to other studies $[2,3,8,9]$. One additional lesson learned in this pilot in situ simulation is the importance of having staff identified to inform bystanders and that this is a training scenario. Thus, we had a patient waiting for an outpatient MRI in the waiting area who became concerned about someone having a cardiac arrest. Although a simulation staff member was able to inform the patient without any negative consequences, in the future we plan to have additional personnel available to mitigate such a situation.

\section{CONCLUSION}

In situ simulation is a valuable tool for the identification of knowledge, skill and system gaps that can allow for targeted training and system redesign to improve patient safety.

Conflict of interest: None declared.

\section{REFERENCES}

1. Issenberg SB, McGaghie WC, Petrusa ER, et al. Features and uses of high-fidelity medical simulations that lead to effective learning: a BEME systematic review. Med Teach. 2005; 27(1): 10-28, doi: 10.1080/01421590500046924, indexed in Pubmed: 16147767.

2. Guise JM, Mladenovic J. In situ simulation: identification of systems issues. Semin Perinatol. 2013; 37(3): 161-165, doi: 10.1053/j.semperi.2013.02.007, indexed in Pubmed: 23721772.

3. Walker ST, Sevdalis N, McKay A, et al. Unannounced in situ simulations: integrating training and clinical practice. BMJ Qual Saf. 2013; 22(6): 453-458, doi: 10.1136/bmjqs-2012-000986, indexed in Pubmed: 23211281.

4. Lopreiato JO, Downing D, Gammon W. Healthcare Simulation Dictionary. 2016. Retrieved from www.ssih.org/dictionary.

5. Failure Modes and Effects Analysis. 2004 Institute for Healthcare Improvement.

6. Patterson MD, Geis GL, Falcone RA, et al. In situ simulation: detection of safety threats and teamwork training in a high risk emergency department. BMJ Qual Saf. 2013; 22(6): 468-477, doi: 10.1136/ bmjqs-2012-000942, indexed in Pubmed: 23258390.

7. 2015 AHA Guidelines for CPR and ECC. https://eccguidelines.heart. org/index.php/circulation/cpr-ecc-guidelines-2/.

8. Zimmermann $\mathrm{K}$, Holzinger IB, Ganassi L, et al. Inter-professional in-situ simulated team and resuscitation training for patient safety: Description and impact of a programmatic approach. BMC Med Educ. 2015; 15: 189, doi: 10.1186/s12909-015-0472-5, indexed in Pubmed: 26511721.

9. Mundell WC, Kennedy CC, Szostek JH, et al. Simulation technology for resuscitation training: a systematic review and meta-analysis. Resuscitation. 2013; 84(9): 1174-1183, doi: 10.1016/j.resuscitation.2013.04.016, indexed in Pubmed: 23624247. 